\title{
04.1;04.3
}

\section{Пылевые звуковые солитоны в запыленной ионосферной плазме, содержащей адиабатически захваченные электроны}

\author{
() С.И. Копнин, С.И. Попель \\ Институт космических исследований РАН, Москва, Россия \\ Национальный исследовательский университет „Высшая школа экономики“, Москва, Россия \\ E-mail: serg_kopnin@mail.ru
}

Поступило в Редакцию 12 апреля 2019г.

В окончательной редакции 12 апреля 2019г.

Принято к публикации 3 июля 2019 г.

Рассмотрена возможность распространения локализованных волновых структур, таких как пылевые звуковые солитоны, в запыленной ионосферной плазме, которая содержит фотоэлектроны, электроны и ионы ионосферы, а также заряженные пылевые частицы. Определены области возможных скоростей и амплитуд солитонов. Найдены солитонные решения для различных размеров и концентраций пылевых частиц в запыленной ионосферной плазме.

Ключевые слова: пылевая плазма, пылевые звуковые солитоны, пылевая звуковая мода, адиабатически захваченные электроны.

DOI: 10.21883/PJTF.2019.20.48389.17837

Когда ионосферная плазма содержит пылевые частицы, говорят о запыленной ионосферной плазме [1]. В ионосферу пылевые частицы могут попадать в результате бомбардировки микрометеоритами (см., например, [2,3]). Пылевые частицы могут возникать в результате конденсации паров воды (см., например, $[4,5])$. Не исключен конвективный перенос частиц из нижних слоев атмосферы [6]. Размеры пылевых частиц в ионосферной плазме обычно могут достигать величин от нескольких единиц до нескольких сотен нанометров с характерными концентрациями в пределах от нескольких десятков до нескольких сотен частиц на кубический сантиметр (см., например, $[7,8]$ ). Если такие частицы содержат примеси щелочно-земельных металлов метеороидного происхождения или целиком состоят из подобного вещества, то в дневное время на поверхности этих частиц может иметь место фотоэффект. В результате фотоэффекта (наряду с воздействием токов электронов и ионов ионосферной плазмы и обратного тока фотоэлектронов) пылевые частицы приобретают существенный положительный заряд [9]. Такие частицы могут играть заметную и даже определяющую роль при описании плазменно-волновых процессов.

В настоящее время проводятся регулярные исследования запыленной ионосферы, и в том числе достаточно интенсивных нелинейных плазменных волновых структур, например, при работе нагревных стендов типа HAARP, Tromso и др. [10]. Таким образом, актуальной становится проблема исследования нелинейных волновых структур в пылевой плазме (см., например, [11-13]), типичными для которой являются пылевые звуковые волны. В настоящей работе рассматриваются нелинейные волновые структуры в запыленной ионосфере, свойственные пылевой плазме, а именно пылевые звуковые солитоны. Обычно при рассмотрении пылевых звуковых нелинейных структур не учитывается захват электронов этими нелинейными структурами. В настоящей работе отмечается важность (по крайней мере для плазмы запыленной ионосферы) адиабатического захвата электронов, что существенно модифицирует параметры нелинейных структур и важно с точки зрения адекватной интерпретации данных имеющихся и будущих наблюдений, а также постановки будущих активных ионосферных экспериментов.

Для описания пылевых звуковых солитонов (см., например, $[14,15])$ можно использовать следующую систему уравнений, включающую в себя уравнение непрерывности и уравнение движения (Эйлера) для пылевых частиц, уравнение Пуассона для самосогласованного электростатического потенциала $\Phi$ в пылевой ионосферной плазме:

$$
\begin{aligned}
\frac{\partial n_{d}}{\partial t}+\frac{\partial\left(n_{d} v_{d}\right)}{d x} & =0, \frac{\partial v_{d}}{d t}+v_{d} \frac{\partial\left(v_{d}\right)}{\partial x}=-\frac{Z_{d} e}{m_{d}} \frac{\partial \Phi}{\partial x}, \\
\frac{\partial^{2} \Phi}{\partial x^{2}} & =4 \pi e\left(n_{e}-n_{i}-n_{d} Z_{d}\right),
\end{aligned}
$$

где пространственная переменная $x$ имеет горизонтальное направление, $n_{e(i, d)}-$ концентрации электронов (ионов, пылевых частиц), $-e-$ заряд электрона, $Z_{d} e$ и $m_{d}$ - заряд и масса пылевой частицы, $v_{d}-$ ее направленная скорость.

Кроме того, необходимо учесть распределения ионов и электронов, которые успевают установиться на пылевых звуковых временны́х масштабах. Обычно рассматриваются распределения Больцмана для электронов и ионов. Однако пылевые звуковые солитоны в рассматриваемых условиях индуцируют положительный электростатический потенциал [16], который представляет 

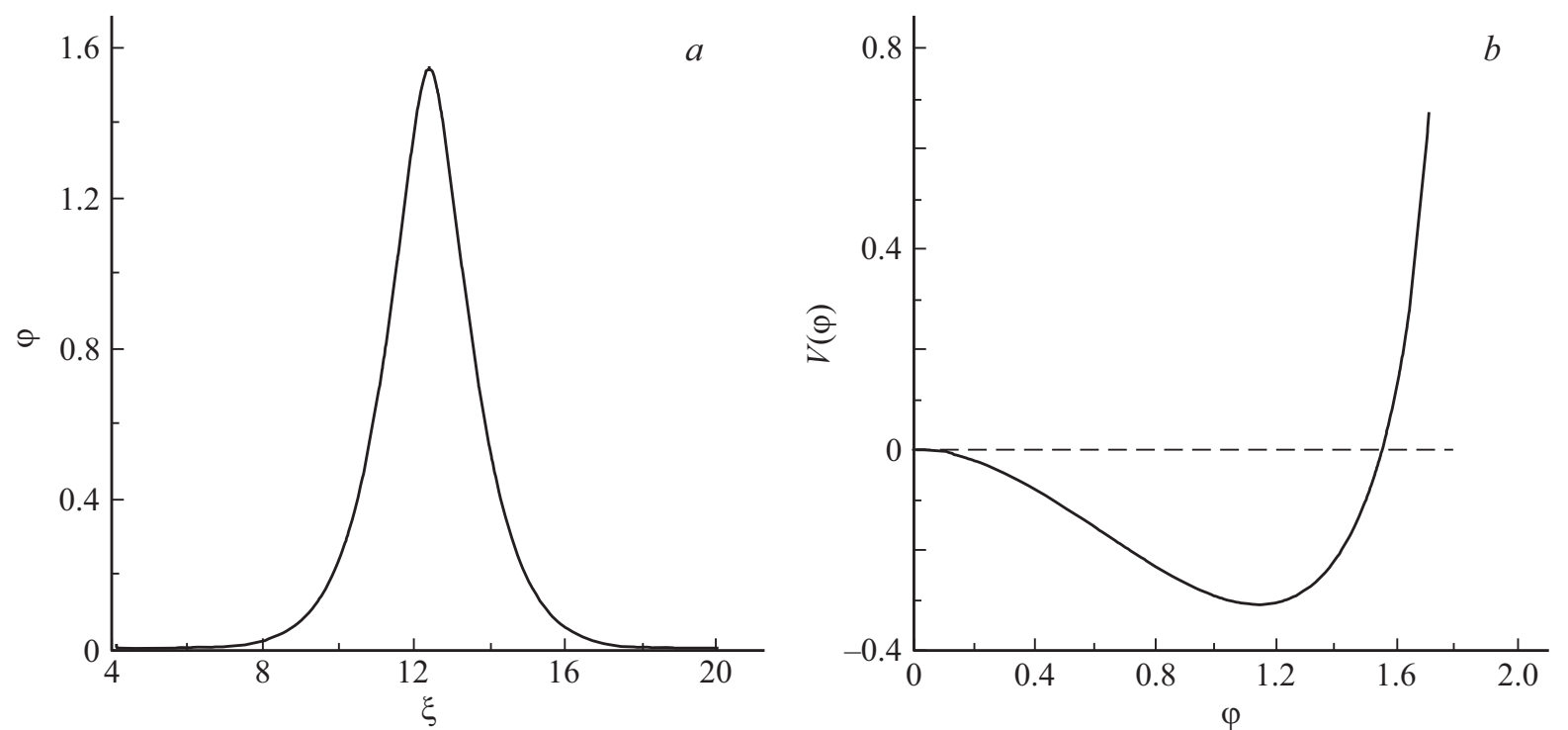

Рис. 1. Характерные виды солитонов $(a)$ и сагдеевских потенциалов $(b)$ для параметров запыленной ионосферной плазмы, соответствующих высотам около $90 \mathrm{~km}$, в случае, когда $a=50 \mathrm{~nm}$.
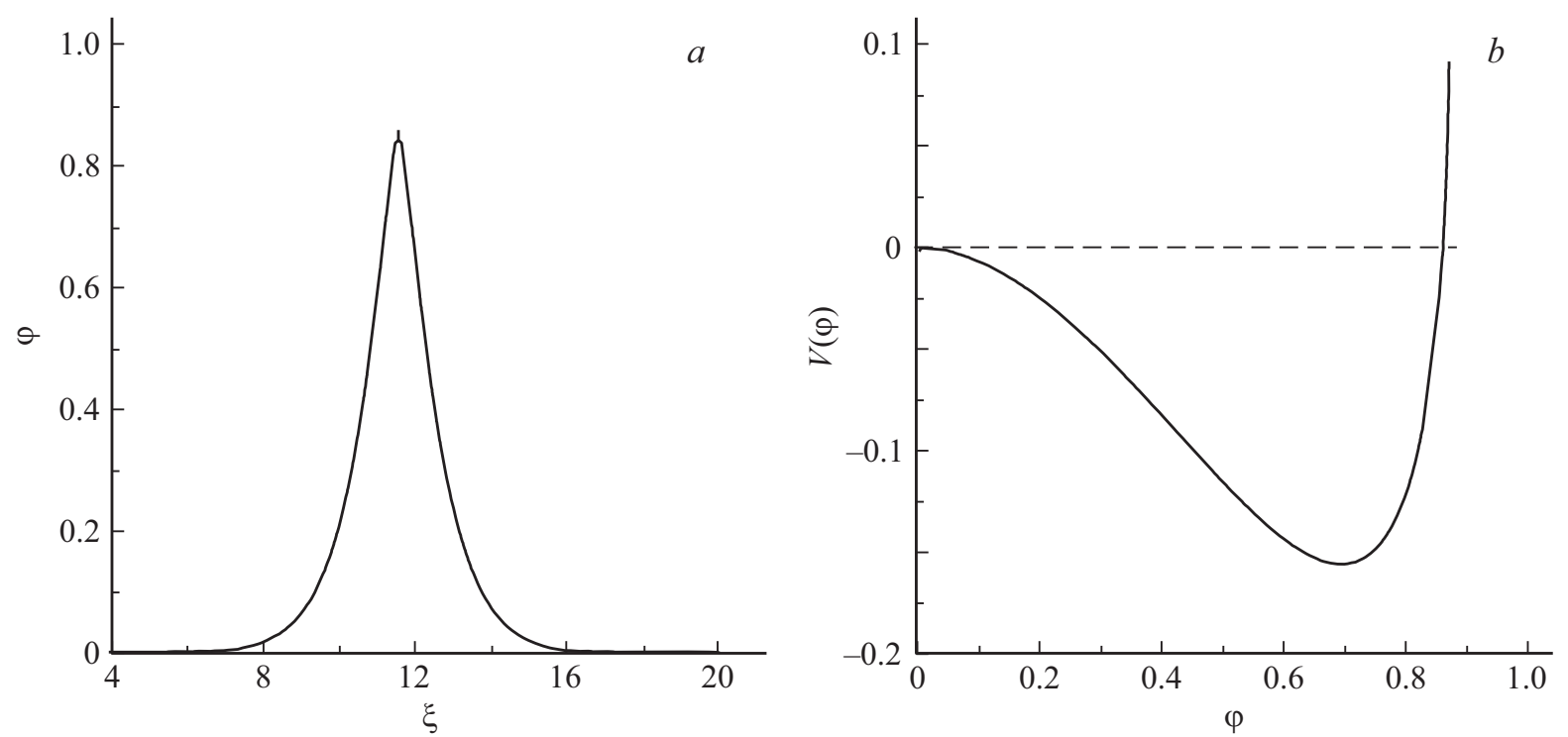

Рис. 2. Характерные виды солитонов $(a)$ и сагдеевских потенциалов $(b)$ для параметров запыленной ионосферной плазмы, соответствующих высотам около $90 \mathrm{~km}$, в случае, когда $a=100 \mathrm{~nm}$.

собой потенциальные стенки для электронов. Распределение Больцмана для электронов можно применять в том случае, когда электроны не захватываются этими потенциальными стенками. Данное условие нарушается, если выполнено следующее неравенство [17]:

$$
\tau_{\text {sol }} \geqslant l_{\text {sol }} / v_{T e},
$$

где $\tau_{s o l}-$ характерное время формирования солитона, $l_{s o l}$ - характерная ширина солитона, $v_{T e}-$ тепловая скорость электронов. Значение $\tau_{s o l}$ порядка $\omega_{p d}^{-1}$ (где $\omega_{p d}$ - пылевая плазменная частота), пространственный масштаб $l_{\text {sol }}$ порядка дебаевского радиуса электронов $\lambda_{\mathrm{D} e}$. Таким образом, $l_{s o l} / v_{T e}=\omega_{p e}^{-1}$ (где $\omega_{p e}-$ плазменная частота электронов). Поэтому неравенство (2) выполнено практически всегда. В этом случае распределение электронов модифицируется благодаря адиабатическому захвату [17] и описывается формулой Гуревича (для ионов подобного не происходит, и оказывается возможным считать распределение ионов больцмановским):

$$
\begin{gathered}
n_{e}=n_{e 0}\left[\left(1-\frac{2}{\sqrt{\pi}} \int_{0}^{\sqrt{e \Phi / T_{e}}} e^{-u^{2}} d u\right) e^{\frac{e \Phi}{T_{e}}}+\frac{2}{\sqrt{\pi}} \sqrt{\frac{e \Phi}{T_{e}}}\right] \\
n_{i}=n_{i 0} e^{-\frac{e \Phi}{T_{i}}}
\end{gathered}
$$



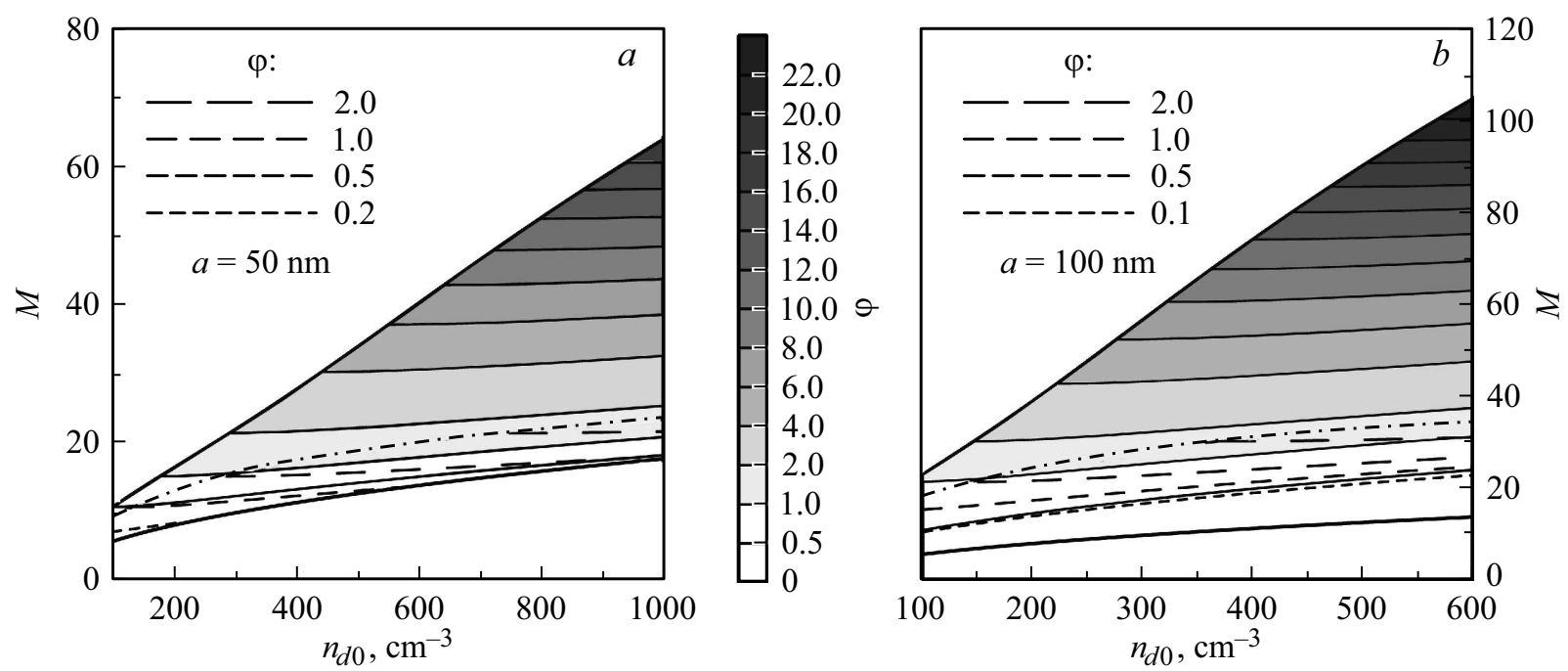

Рис. 3. Профили амплитуд для пылевых звуковых солитонов в ситуации, учитывающей адиабатический захват электронов, на плоскости $\left(M, n_{d 0}\right)$ в случаях, когда средний размер пылевых частиц равен $50(a)$ и $100 \mathrm{~nm}(b)$. Штриховыми изолиниями показаны амплитуды солитонов в случаях, когда электроны подчинены распределению Больцмана (при этом нижняя граница области определения совпадает с аналогичной границей в ситуации, учитывающей адиабатический захват электронов, а верхняя граница отмечена штрихпунктирной линией).

Здесь $T_{e(i)}$ - температура электронов (ионов), выраженная в энергетических единицах, индекс 0 соответствует невозмущенным состояниям.

Методом сагдеевского потенциала из системы уравнений (1)-(3) можно получить локализованные волновые решения, распространяющиеся с некоторой постоянной скоростью $V$. Таким образом, все параметры задачи должны зависеть от координаты $x$ и времени $t$ посредством только переменной $\eta=x-V t$. Вводя безразмерные переменные $e \Phi / T_{e} \rightarrow \varphi, V / C_{S d} \rightarrow M, \eta / \lambda_{\mathrm{D} e} \rightarrow \xi$, где $C_{S d}=\sqrt{T_{e} / m_{d}}, \lambda_{\mathrm{De}}=\sqrt{T_{e} / 4 \pi n_{e 0} e^{2}}$, можно выписать выражение, описывающее сагдеевский потенциал:

$$
\begin{gathered}
V(\varphi)=1-e^{\varphi}\left(1-\frac{2}{\sqrt{\pi}} \int_{0}^{\sqrt{\varphi}} e^{-u^{2}} d u\right) \\
-\frac{2}{\sqrt{\pi}} \sqrt{\varphi}-\frac{4}{3} \frac{\varphi^{3 / 2}}{\sqrt{\pi}}+\frac{1-e^{-\varphi \tau_{i}}}{\tau_{i}} \\
+\frac{Z_{d} d}{\tau_{t}}\left[1-e^{\varphi \tau_{i}}\left(1-\frac{2}{\sqrt{\pi}} \int_{0}^{\sqrt{\varphi \tau_{t}}} e^{-u^{2}} d u\right)\right. \\
\left.-\frac{2}{\sqrt{\pi}} \sqrt{\varphi \tau_{t}}-\frac{4}{3} \frac{\left(\varphi \tau_{t}\right)^{3 / 2}}{\sqrt{\pi}}\right]+M d\left(M-\sqrt{M^{2}-2 Z_{d} \varphi}\right)
\end{gathered}
$$

где $d=n_{d 0} / n_{e 0}, \tau_{t}=T_{e} / T_{e t}, \tau_{i}=T_{e} / T_{i}$. Область определения $\left(M_{\min }<M<M_{\max }\right)$ для локализованных волновых решений определяется условиями их существования и имеет вид

$$
M^{2} \geqslant M_{\min }^{2}=\frac{Z_{d} d}{1+\tau_{i}+Z_{d} d \tau_{t}},
$$

$$
\begin{aligned}
& 1-e^{\frac{M_{\max }^{2}}{2 Z_{d}}}\left(1-\frac{2}{\sqrt{\pi}} \int_{0}^{\sqrt{M_{\max }^{2} / 2 Z_{d}}} e^{-u^{2}} d u\right)-\frac{2}{\sqrt{\pi}} \sqrt{\frac{M_{\max }^{2}}{2 Z_{d}}} \\
& -\frac{4}{3} \frac{\left(M_{\max }^{2} / 2 Z_{d}\right)^{3 / 2}}{\sqrt{\pi}}+\frac{1-e^{-\left(M_{\max }^{2} / 2 Z_{d}\right) \tau_{i}}}{\tau_{i}} \\
& +\frac{Z_{d} d}{\tau_{t}}\left[1-e^{\frac{M_{\max }^{2}}{2 Z_{d}}}\left(1-\frac{2}{\sqrt{\pi}} \int_{0}^{\sqrt{\left(\left(M_{\max }^{2} / 2 Z_{d}\right) \tau_{t}\right)}} e^{\left.-u^{2} d u\right)}\right.\right. \\
& \left.-\frac{2}{\sqrt{\pi}} \sqrt{\frac{M_{\max }^{2}}{2 Z_{d}} \tau_{t}}-\frac{4}{3} \frac{\left(\left(M_{\max }^{2} / 2 Z_{d}\right) \tau_{t}\right)^{3 / 2}}{\sqrt{\pi}}\right]+M_{\max }^{2} d \geqslant 0 .
\end{aligned}
$$

На рис. 1 и 2 представлены характерные виды солитонов $(a)$ и сагдеевских потенциалов $(b)$ для параметров запыленной ионосферной плазмы, соответствующих высотам около $90 \mathrm{~km} \quad\left(n_{e 0}=n_{i 0}=3 \cdot 10^{4} \mathrm{~cm}^{-3}\right.$, $\left.T_{e 0}=T_{i 0}=141 \mathrm{~K}\right)$. Рис. 1 построен для $a=50 \mathrm{~nm}$ и $n_{d 0}=500 \mathrm{~cm}^{-3}$, а рис. 2 - для $a=100 \mathrm{~nm} \mathrm{и}$ $n_{d 0}=300 \mathrm{~cm}^{-3}$.

На рис. 3 представлены профили амплитуд для пылевых звуковых солитонов на плоскости $\left(M, n_{d 0}\right)$ с учетом адиабатического захвата электронов в случаях, когда средний размер пылевых частиц равен 50 (a) и $100 \mathrm{~nm}$ $(b)$. Также на этих рисунках штриховыми изолиниями показаны амплитуды солитонов в случаях, когда электроны подчинены распределению Больцмана (адиабатический захват не учитывается). Видно, что в этом случае области определения солитонов значительно меньше, чем в аналогичном случае, учитывающем адиабатиче- 
ский захват электронов: нижняя граница для чисел Маха не изменяется, а верхняя расположена значительно ниже (на рисунках отмечена штрихпунктирной линией). Можно также отметить, что амплитуды солитонов для соответствующих чисел Маха в случае, учитывающем адиабатический захват электронов, заметно меньше амплитуд в ситуации, когда электроны распределены в соответствии с распределением Больцмана. Кроме того, в случае с распределением Гуревича существуют решения (для больших чисел Маха) с заметно бо́льшими амплитудами солитонов в тех областях определения, где в случае с распределением Больцмана решений уже нет.

Видно, что во всей области определения амплитуды пылевых звуковых солитонов оказываются того же знака, что и заряд пылевых частиц (при $Z_{d} e>0$ амплитуда солитона $\left.\varphi_{0}>0\right)$.

В работе рассмотрена возможность распространения локализованных волновых структур, таких как пылевые звуковые солитоны, в запыленной ионосферной плазме, которая включает в себя фотоэлектроны, электроны и ионы ионосферы, а также заряженные пылевые частицы. Показано, что при рассмотрении волновых процессов следует учитывать эффект захвата электронов потенциальными стенками, существование которых обусловлено положительным электростатическим потенциалом солитона. Определены области возможных скоростей и амплитуд солитонов. Найдены солитонные решения для различных размеров и концентраций пылевых частиц в запыленной ионосферной плазме.

\section{Конфликт интересов}

Авторы заявляют, что у них нет конфликта интересов.

\section{Список литературы}

[1] Клумов Б.А., Морфилл Г.Е., Попель С.И. // ЖЭТФ. 2005. T. 127. B. 1. C. $171-185$.

[2] Hunten D.M., Turco R.P., Toon O.B. // J. Atmos. Sci. 1980. V. 37. P. $1342-1357$.

[3] Мусатенко С.И., Мусатенко Ю.С., Курочка Е.В., Ласточкин А.В., Чолий В.Я., Максименко О.И., Слипченко А.C. // Геомагнетизм и аэрономия. 2006. Т. 46. № 2. C. $182-192$.

[4] Havnes O., de Angelis U., Bingham R., Goertz C.K., Morfill G.E., Tsytovich V.N. // J. Atmos. Terr. Phys. 1990. V. 52. P. 637-643.

[5] Бронштэн B.A. Серебристые облака и их наблюдение. М.: Наука, 1984. $128 \mathrm{c.}$

[6] Rao N.N., Shukla P.K., Yu. M.Y. // Planet. Space Sci. 1990. V. 35. P. 543-546.

[7] Копнин С.И., Косарев И.Н., Попель С.И., Минг Ю. // Физика плазмы. 2005. Т. 31. № 2. С. 224-232.

[8] Копнин С.И., Попель С.И., Минг Ю. // Физика плазмы. 2007. T. 33. № 4. С. 323-336.

[9] Копнин С.И., Моржсакова А.А., Попель С.И., Шукла П.К. // Физика плазмы. 2011. Т. 37. № 8. С. 745-755.
[10] Борисов Н.Д., Копнин С.И., Морозова Т.И., Попель С.И. // Физика плазмы. 2019. Т. 45. № 4. С. 346-352.

[11] Медведев Ю.В. // Физика плазмы. 2019. Т. 45. № 3. С. 250 257.

[12] Borah P., Das N. // Plasma Phys. Rep. 2018. V. 44. P. 738 745.

[13] El-Labany S.K., El-Bedwehy N.A., Selim M.M., AlAbbasy O.M. // Phys. Plasmas. 2015. V. 22. P. 023711. https://doi.org/10.1063/1.4913649

[14] Kopnin S.I., Kosarev I.N., Popel S.I., Yu M.Y. // Planet. Space Sci. 2004. V. 52. P. 1187-1194.

[15] Popel S.I., Kopnin S.I., Kosarev I.N., Yu M.Y. // Adv. Space Res. 2006. V. 37. P. 414-419.

[16] Морозова Т.И., Копнин С.И., Попель С.И. // Физика плазмы. 2015. Т. 41. № 10. С. 867-876.

[17] Lifshitz E.M., Pitaevskii L.P. Physical Kinetics. Oxford: Pergamon Press, 1981. P. 146. 